\title{
Timing of blood sampling for butyrylcholinesterase (BChE) phenotyping in patients with prolonged recovery after mivacurium or suxamethonium.
}

\author{
N. Mintjens ${ }^{1}$, R. Brummans ${ }^{2}$, F. Soetens ${ }^{2}$, K. Claes $^{3}$, L. Vanlinthout ${ }^{4}$ \\ ${ }^{1}$ Dept of anaesthesia, University Hospital Antwerp - Edegem (Belgium), ${ }^{2}$ Dept of anaesthesia, AZ Turnhout - Turnhout (Belgium), \\ ${ }^{3}$ Centre for Medical Genetics, Ghent University Hospital - Ghent (Belgium), ${ }^{4}$ Dept of anaesthesia, GZA Hospitals - Antwerp (Belgium)
}

Background and Goal of Study: About $76 \%$ of the population is homozygous for usual BChE, while $24 \%$ carries at least one genetic variant allele. Most of these variants have reduced $\mathrm{BChE}$ activity ([BChE]) that can lead to prolonged neuromuscular block (NMB) after suxamethonium (SUX) or mivacurium (MIV). BChE can be characterized by phenotyping and by determination of genotype. Inappropriate timing of blood sampling can interfere with results of BChE assay. However, guidelines regarding desirable delay between exposure to anaesthesia/neuromuscular blocker and testing are not clearly defined. We therefore compared BChE phenotype based on blood samples collected during or shortly $(<6 \mathrm{~h})$ after recovery from NMB (T1) with that assessed on blood drawn after $>1$ week (T2).

Materials and Methods: After ethical committee approval and written informed consent 19 patients with a prolonged NMB after MIV and SUX were selected. Screening at T1 and T2 was done in all patients. [BChE] was assayed using benzoylcholine as a substrate. Phenotyping was based on dibucaine and fluoride inhibition. Genotyping was done by the Centre of Medical Genetics (University of Ghent). [BChE] at T1 and T2 were compared using a paired t-test. The effect of neostigmine on [BChE] was assessed by analysis of co-variance.

Results and Discussion: We included 19 patients with prolonged NMB following MIV (18) and SUX (1). MIV dose was $0.17(0.1) \mathrm{mg} \cdot \mathrm{kg}^{-1}$ and NMB duration (TOF90) was 184 (42) min. [BChE] were significantly different at T1 and T2, i.e. 2459 (1905) U. $\mathrm{L}^{-1}$ and $4287(2636) \mathrm{U} \cdot \mathrm{L}^{-1}$, respectively $(\mathrm{p}<0.002)$. Values are mean (SD). Neostigmine did not significantly decrease [BChE] at T1. In 3 patients at $\mathrm{T} 1$ and 2 patients at T2, [BChE] was too low to determine phenotype. In all other patients phenotyping yielded identical results at T1 and T2. The presumed presence of at least one allele for the atypical variant on phenotypic characterization was confirmed by sequence analysis of patient's DNA. Phenotyping failed to identify one new variant (p.Tyr146Cys) and in detecting the $K$ variant in 14 out of 15 patients.
Conclusion(s): Anaesthesia interfered with [BChE], but not with phenotyping. Phenotyping can be performed on blood drawn during or immediately after recovery of NMB to screen for clinically relevant variants of BChE. However, accurate diagnosis of BChE deficiency needs further confirmation by determination of genotype [1].

\begin{tabular}{|c|c|c|c|c|c|}
\hline \multirow[t]{2}{*}{ Patient } & \multicolumn{2}{|c|}{ Phenotype } & \multicolumn{3}{|c|}{ Genotype } \\
\hline & T1 & T2 & $\begin{array}{c}\text { A- } \\
\text { variant }\end{array}$ & $\begin{array}{c}\text { K- } \\
\text { variant }\end{array}$ & Other \\
\hline 1 & $\mathbf{U}, \mathbf{U}$ & $\mathbf{U}, \mathbf{U}$ & $-/-$ & $-/-$ & \\
\hline 2 & $\mathbf{A}, \mathbf{A}$ & $\mathbf{A}, \mathbf{A}$ & $+/-$ & $+/-$ & \\
\hline 3 & $\mathbf{U}, \mathbf{A}$ & U,A & $+/-$ & $+/+$ & \\
\hline 4 & $\mathbf{A}, \mathbf{A}$ & $\mathbf{A}, \mathbf{A}$ & $+/+$ & $+/+$ & \\
\hline 5 & Nm & U,A & $-/-$ & $+/-$ & $\mathbf{F 1}, \S$ \\
\hline 6 & $A, K$ & $A, K$ & $+/-$ & $+/+$ & \\
\hline 7 & U,A & U,A & $+/-$ & $+/+$ & \\
\hline 8 & $\mathbf{U}, \mathbf{U}$ & Nm & $-/-$ & $+/+$ & \\
\hline 9 & $\mathbf{A}, \mathbf{A}$ & $\mathbf{A}, \mathbf{A}$ & $+/+$ & $+/+$ & \\
\hline 10 & $\mathbf{U}, \mathbf{A}$ & $\mathbf{U}, \mathbf{A}$ & $+/-$ & $+/-$ & \\
\hline 11 & Nm & Nm & $+/+$ & $+/+$ & \\
\hline 12 & $\mathbf{U}, \mathbf{U}$ & $\mathbf{U}, \mathbf{U}$ & $-/-$ & $-/-$ & \\
\hline 13 & $\mathbf{A}, \mathbf{A}$ & $\mathbf{A}, \mathbf{A}$ & $+/+$ & $+/+$ & \\
\hline 14 & Nm & $\mathbf{U}, \mathbf{U}$ & $-/-$ & $+/-$ & \\
\hline 15 & $\mathbf{N a}$ & $\mathbf{U}, \mathbf{U}$ & $-/-$ & $-/-$ & \\
\hline 16 & $\mathbf{A}, \mathbf{A}$ & $\mathbf{A}, \mathbf{A}$ & $+/+$ & $+/+$ & \\
\hline 17 & $\mathbf{U}, \mathbf{A}$ & $\mathbf{U}, \mathbf{A}$ & $+/-$ & $+/-$ & \\
\hline 18 & $\mathbf{A}, \mathbf{K}$ or $\mathbf{A}, \mathbf{J}$ & $\mathbf{A}, \mathbf{K}$ or $\mathbf{A}, \mathbf{J}$ & $+/+$ & $+/-$ & \\
\hline 19 & $\mathbf{U}, \mathbf{U}$ & $\mathbf{U}, \mathbf{U}$ & -/- & -/- & \\
\hline
\end{tabular}

Table 1. Phenotype (at T1 and T2) and genotype of BChE. $\mathrm{Nm}$ : not measurable; Na: not available

-/-: no variant; +/-: heterozygote; +/+: homozygote. $\S$ : new variant of BChE; p.Tyr146Cys. 